\title{
Effect of suprathermal electrons on the intensity and Doppler frequency of electron plasma lines
}

\author{
P. Guio ${ }^{1}$, J. Lilensten ${ }^{2}$ \\ ${ }^{1}$ The Auroral Observatory, Institute of Physics, University of Tromsø, Norway \\ ${ }^{2}$ Laboratoire de planétologie de Grenoble, LIS-ENSIEG BP 46, F-38402 Saint-Martin-d'Hères, France
}

Received: 2 November 1998 / Revised: 10 February 1999 / Accepted: 11 February 1999

\begin{abstract}
In an incoherent scattering radar experiment, the spectral measurement of the so-called up- and downshifted electron plasma lines provides information about their intensity and their Doppler frequency. These two spectral lines correspond, in the backscatter geometry, to two Langmuir waves travelling towards and away from the radar. In the daytime ionosphere, the presence of a small percentage of photoelectrons produced by the solar EUV of the total electron population can excite or damp these Langmuir waves above the thermal equilibrium, resulting in an enhancement of the intensity of the lines above the thermal level. The presence of photo-electrons also modifies the dielectric response function of the plasma from the Maxwellian and thus influences the Doppler frequency of the plasma lines. In this paper, we present a high time-resolution plasma-line data set collected on the EISCAT VHF radar. The analysed data are compared with a model that includes the effect of a suprathermal electron population calculated by a transport code. By comparing the intensity of the analysed plasma lines data to our model, we show that two sharp peaks in the electron suprathermal distribution in the energy range 20-30 eV causes an increased Landau damping around $24.25 \mathrm{eV}$ and $26.25 \mathrm{eV}$. We have identified these two sharp peaks as the effect of the photoionisation of $\mathrm{N}_{2}$ and $\mathrm{O}$ by the intense flux of monochromatic HeII radiation of wavelength $30.378 \mathrm{~nm}(40.812 \mathrm{eV})$ created in the chromospheric network and coronal holes. Furthermore, we see that what would have been interpreted as a mean Doppler drift velocity for a Maxwellian plasma is actually a shift of the Doppler frequency of the plasma lines due to suprathermal electrons.
\end{abstract}

Key words. Ionosphere (electric fields and currents; solar radiation and cosmic ray effects).

Correspondence to: P. Guio

e-mail: patrick@phys.uit.no

\section{Introduction}

The enhancement process of the plasma line is well understood theoretically (Perkins and Salpeter, 1965; Yngvesson and Perkins, 1968; Bjørnå et al., 1982; Bjørnå and Trulsen, 1986) and has been confirmed by measurements both during daytime (Perkins et al., 1965; Yngvesson and Perkins, 1968; Lejeune and Kofman, 1977; Kofman and Lejeune, 1980) and in auroral conditions (Oran et al., 1981; Kirkwood et al., 1995). The problem of estimating the field-aligned electron net current from an incoherent scatter Doppler measurement of the electron plasma lines has also been studied for some time (Vidal-Madjar et al., 1975; Bauer et al., 1976; Showen, 1979), yet the work on the subject has progressed rather slowly, partly due to experimental problems (the spectra needs to be estimated) and partly due to the lack of theoretical understanding. It is expected that by simultaneous measurements of the frequency of the up- and downshifted plasma lines one could deduce the mean Doppler velocity $u_{e}$ of the electron velocity distribution function along the scattering direction.

At first, an additional asymmetry between the Doppler frequency of the plasma lines was identified for an isotropic Maxwellian plasma. This asymmetry is due to the difference in magnitude of the wave numbers of the up- and down-going Langmuir waves (Showen, 1979). This term is proportional to a temperaturedependent correction term (Debye correction) in the dispersion relation for Langmuir waves and was pointed out as a possible method for the independent determination of the electron temperature (Hagfors and Lehtinen, 1981). Later, Kofman et al. (1993) showed that by introducing in the dispersion relation a heat flow correction term that takes into account the effect of the temperature gradient of the electron temperature, that their data collected with the EISCAT UHF radar during daytime were in better agreement with the 
prediction of no field-aligned. This heat flow correction term behaves as an additional mean Doppler velocity that artificially increases the difference of the Doppler shifts of the plasma lines. Guio et al. (1998) showed that the heat flow effect was underestimated for UHF radars by comparing the heat flow approximation of the dispersion relation with a full numerical estimation of the dielectric response function using the Spitzer velocity distribution for a plasma with a temperature gradient (Cohen et al., 1950; Spitzer and Härm, 1953). It was also shown that for VHF radars, suprathermal electrons would contribute to modify the dielectric response function and thus the Doppler frequency of the plasma lines. It is noteworthy that while the suprathermal effect on the Doppler frequency has been investigated with a Maxwellian to represent the photoelectrons distribution (Bauer et al., 1976), it has to our knowledge, never been observed in data.

In this paper we present a high temporal resolution data set collected on the EISCAT VHF radar. The plasma line analysis consists of two parts. First, the result of the analysis of the intensity of the plasma lines is compared with a model that calculates the theoretical enhancement caused by suprathermal electrons described by the angular velocity distribution derived form the electron angular intensity flux calculated by a transport code (Lummerzheim, 1987; Lummerzheim and Lilensten, 1994). Once we find an electron velocity distribution that gives correct intensity compared to the measured intensity, we use the full numerical calculation of the plasma dielectric response (Guio et al., 1998) to estimate the mean Doppler velocity $u_{e}$ of the ambient electrons, and the plasma frequency $\omega_{e}$. The results are compared with the Maxwellian case.

\section{The data}

The plasma line data were collected with the EISCAT VHF radar $(223.8 \mathrm{MHz})$ near Tromsø, Norway on 22 June 1994 from 15:00 UT to 16:00 UT. The antenna was pointed vertically which gives an angle of about $12^{\circ}$ to the magnetic field line at $250 \mathrm{~km}$. The ionospheric conditions were quiet, a F10.7 index of 75 and an $A_{p}$ index of 6 were measured.

The data were collected with the long pulse technique first described by Showen (1979) and the measurement of the plasma lines was done at the peak of the F-layer. A special tracking program that interacts with the radar, attempts to tune the radar receiver every dump so that the critical frequency of the peak of the F-layer is centred in the $100 \mathrm{kHz}$ observation window filtered with a $80 \mathrm{kHz}$ filter. Three receiving channels, one for the upshifted, one for the downshifted plasma line and one for the ion line, were used simultaneously, with the same spatial resolution. A single long pulse with length $450 \mu \mathrm{s}$ was transmitted and signal was collected at a sampling rate of $10 \mu \mathrm{s}$. A 33-point autocorrelation function with correlation time between 0 and $320 \mu$ s by step of $10 \mu$ s was computed for five gates as a function of altitude, on each channel. The first gate was situated at $178 \mathrm{~km}$, the gate separation was $37.5 \mathrm{~km}$, thus the last gate is situated at $328 \mathrm{~km}$, and the volume probed for one gate was about $45 \mathrm{~km}$. In addition to the $450 \mu$ s long pulse, a very long pulse of $900 \mu \mathrm{s}$ was transmitted to measure the ion line. Eleven gates were computed from $317 \mathrm{~km}$ up to $1217 \mathrm{~km}$ and the volume probed for one gate was about $100 \mathrm{~km}$. The initial integration period was $2 \mathrm{~s}$, and the up- and downshifted plasma lines autocorrelation functions were analysed at the same time resolution while the ion line data were post-integrated to a 30 -s period before analysis.

Figure 1 presents the ionospheric parameters extracted from the ion line analysis as a function of altitude. Each panel presents the parameter obtained from both the $450 \mu$ s and $900 \mu$ s pulses. The two dashed lines on each panel represent the 1- $\sigma$ deviation of the parameter averaged over the 1-h period while the solid line is the averaged parameter. The fitting procedure did not converge for the $900 \mu \mathrm{s}$ very long pulse above the altitude of $850 \mathrm{~km}$ due to low backscattered signal, as it is seen on the electron density $n_{e}$, the electron and ion temperature $T_{e}$ and $T_{i}$ and the ion mean velocity $u_{i}$. The ionospheric parameters remained very stable over the time period of the experience.

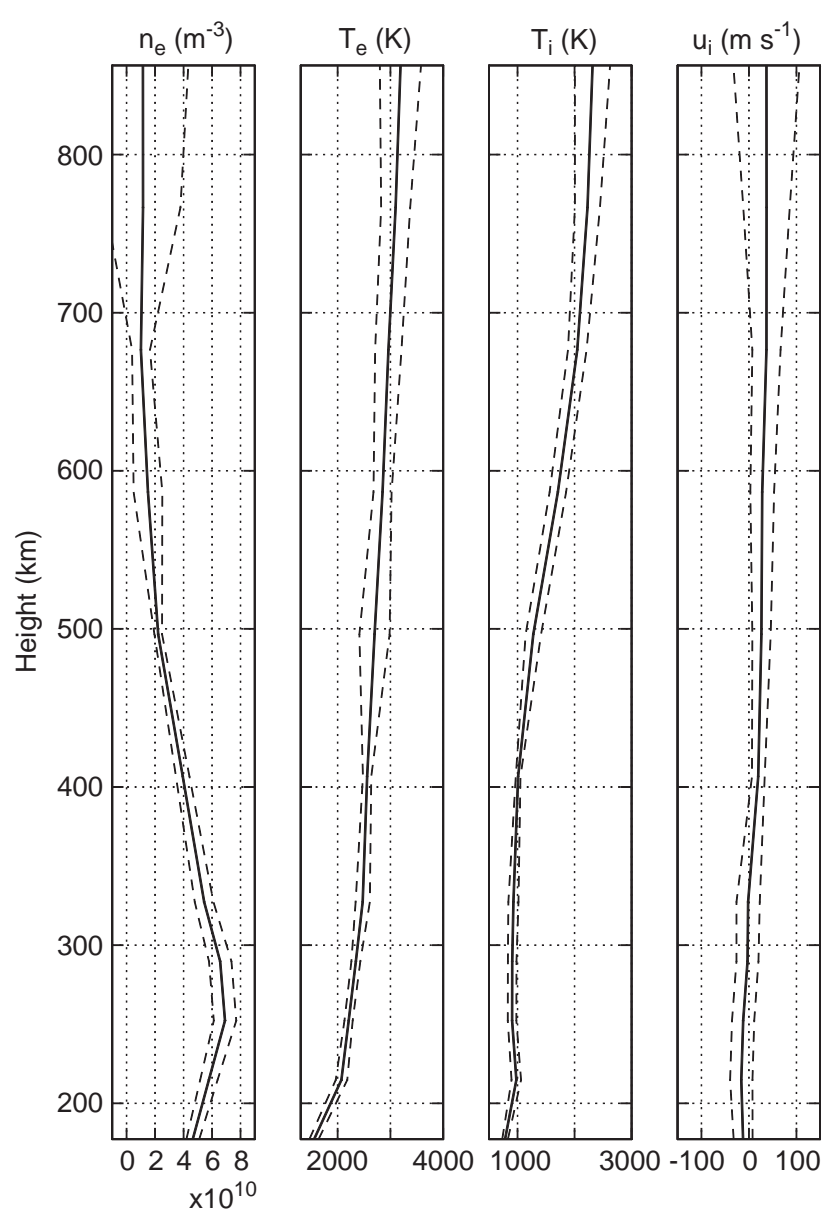

Fig. 1. The electron density $n_{e}$, the electron and ion line temperatures $T_{e}$ and $T_{i}$ and ion mean drift velocity $u_{i}$ as a function of altitude averaged over the 1-h period of the experiment run. The dashed lines represents the 1- $\sigma$ deviation from the averaged parameter (solid line) 
The plasma line data were analysed using a leastsquare fit method that uses a model of the autocorrelation function adapted to the observation of plasma lines with the long pulse technique (Kofman et al., 1981; Heinselman and Vickrey, 1992; Kofman et al., 1993). The least-square fit method allows accurate extraction of the critical frequency at the electron density peak of the F-layer, the intensity and the frequency width of the plasma lines as well as the electron density scale height around the peak and the height of the F-region peak. Figure 2 shows an example of a fit of an autocorrelation function collected at a 2-s integration time. The intensity of the plasma line is fitted in terms of the antenna temperature and is then converted into electron plasma temperature (Yngvesson and Perkins, 1968) by taking

\section{ECHOV 94-06-22 15:09:06 (2s)}

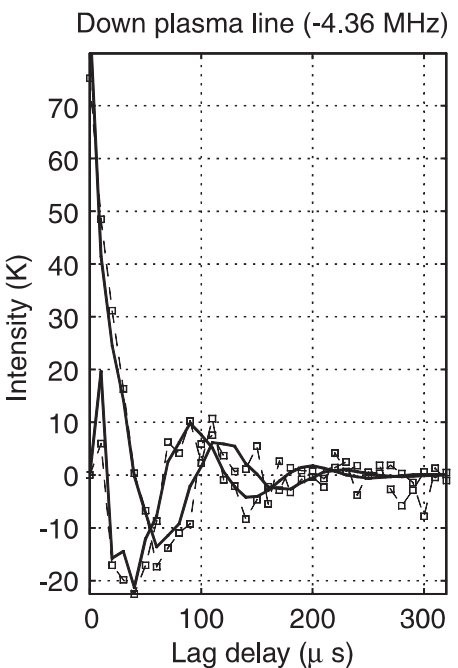

Up plasma line $(+4.36 \mathrm{MHz})$
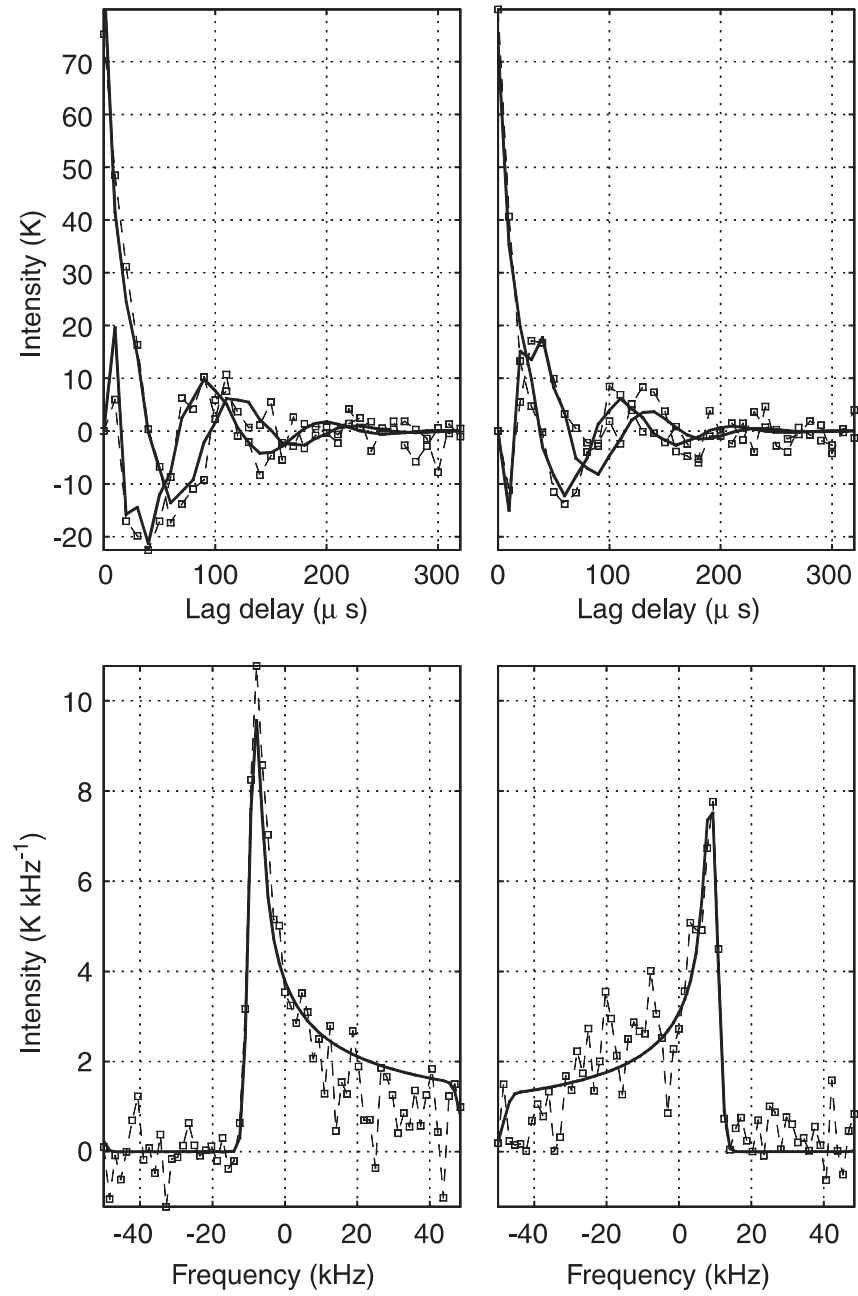

Fig. 2. Example of the result of a fit of the up- and downshifted plasma lines. The two upper panels show the measured complex autocorrelation functions expressed in units of antenna temperature (dashed line) and the theoretical model (solid line). The curves with the intensity equal to zero at the zero lag delay are the imaginary parts. The two lower panels present the corresponding power density spectrum where the critical frequency is more easily identified in the spectral shape into account the radar constant and the frequencydependent effective antenna area (Guio et al., 1996).

Figures 3 and 4 show the parameters extracted from the plasma line analysis, Fig. 3 is for the downshifted plasma line and Fig. 4 for the upshifted. The top panels present the critical frequencies $f_{r \pm}$ and the third panels the intensities of the lines $T_{p \pm}$. The second panels show the corresponding phase energies $E_{\phi \pm}$ of the Langmuir waves at the frequencies $f_{r \pm}$ defined by

$E_{\phi \pm}=\frac{1}{2} m_{e} v_{\phi \pm}^{2}$

where $v_{\phi \pm}=2 \pi f_{r \pm} / k_{ \pm}$is the phase velocity of the wave, $k_{ \pm}=2 \pi\left(f_{0}+f_{0}+f_{r_{ \pm}}\right) / c$ is the scattered wave vector of the Langmuir wave in the backscatter geometry with a radar of frequency $f_{0}$, and $m_{e}$ is the electron mass. The lowest panel in each figure shows the bandwidth $\delta f_{ \pm}$of the plasma lines over the 1-h period of observation. Note that for low-frequency radars such as the EISCAT VHF radar, the difference $\Delta f_{r \pm}=f_{r+}+f_{r-}$ of Doppler frequency between up- and downshifted plasma lines is of the order of $1 \mathrm{kHz}$ and it corresponds in term of
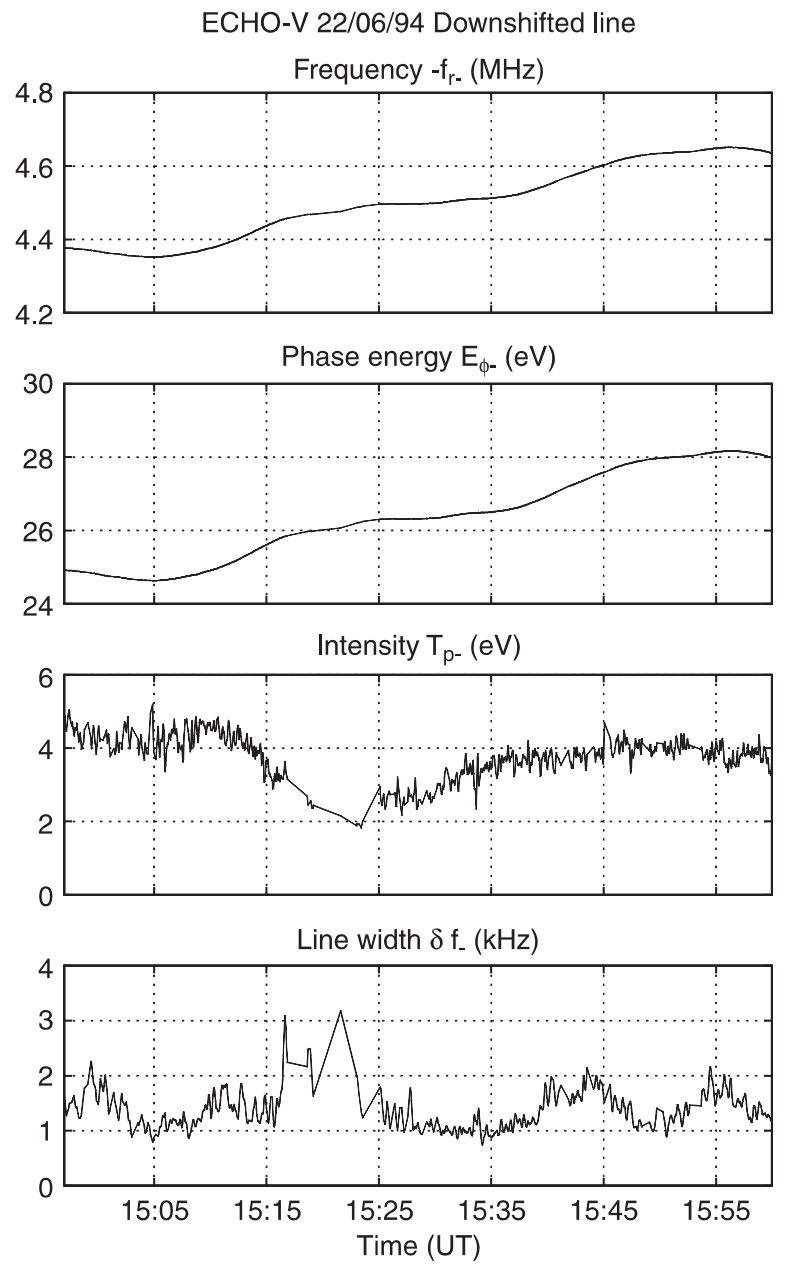

Fig. 3. The parameters extracted from the downshifted plasma line analysis for the 1-h period of observation. From top to bottom: the absolute value of the critical frequency $\left|f_{r-}\right|$, the corresponding phase energy $E_{\phi-}$, the intensity of the line (or plasma line temperature) $T_{p-}$ and the line width $\delta f_{-}$ 
ECHO-V 22/06/94 Upshifted line
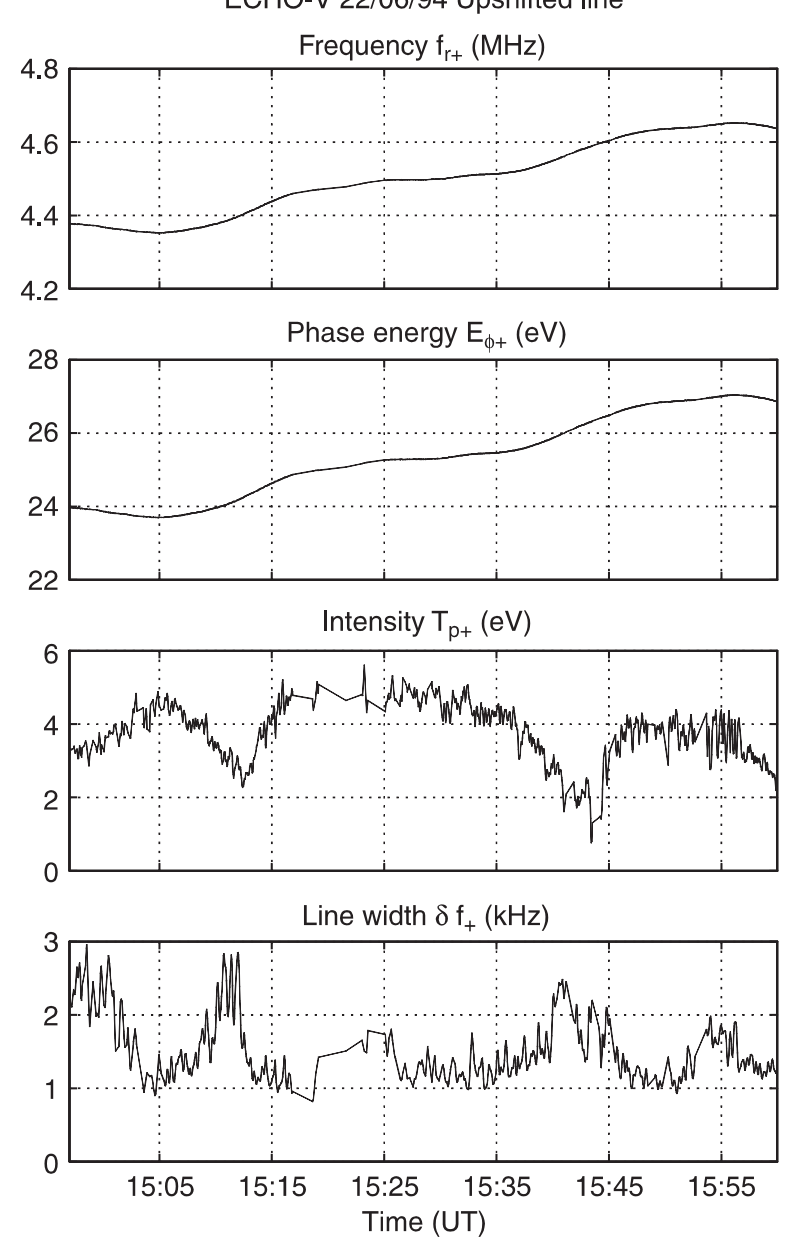

Fig. 4. The parameters extracted from the upshifted plasma line analysis for the 1-h period of observation. From top to bottom: the critical frequency $f_{r+}$, the corresponding phase energy $E_{\phi+}$, the intensity of the line (or plasma line temperature) $T_{p+}$ and the line width $\delta f_{+}$

phase energy into a difference $\Delta E_{\phi \pm}=E_{\phi+}-E_{\phi-}$ of the order of $-1 \mathrm{eV}$.

The intensity of the plasma lines presents interesting variations in the $1-\mathrm{h}$ observation period. At a first look, the intensity of the up- and downshifted line seems to have a very different behaviour as a function of time. In Fig. 4, the intensity of the upshifted plasma line around 15:12 UT, for phase energies $E_{\phi+}$ about $24.25 \mathrm{eV}$ shows a significant drop. A similar drop can be observed around 15:43 UT, for phase energies around $26.25 \mathrm{eV}$. In Fig. 3 the intensity of the downshifted plasma line around 15:24 UT, for phase energies $E_{\phi-}$ around $26.25 \mathrm{eV}$ exhibits also a similar drop. At the same time, the frequency width of the plasma lines is increasing (see the lowest panel of Figs. 3 and 4). For the VHF radar, the natural frequency width of the plasma lines is controlled by the collisional damping and the Landau damping of the suprathermal electrons. The collisional damping is a function of the electron-neutral and electron-ion collision frequencies (Newman and Oran, 1981) and of the phase velocity of the wave (Perkins and Salpeter, 1965). As seen in the third panel from the top in Fig. 6, the electron collision frequency derived from the ion line analysis, does not vary much over the observation period. The phase energy of the plasma lines, as seen in the second panel from top in Figs. 3 and 4 , and thus the phase velocity is increasing smoothly. It is therefore very unlikely that the collisional damping could provoke such variations in the intensity, instead it is likely that the variations in the intensity of the plasma lines are caused by an increase in the Landau damping of the suprathermal electron population at these phase energies (Perkins and Salpeter, 1965). This explanation is reinforced by the fact that both the up- and downshifted plasma lines exhibit a drop in intensity for the same phase energy of $26.25 \mathrm{eV}$, i.e. for the same velocity in the electron velocity distribution. Note that the drop of intensity observed on the downshifted line at 15:24 UT is spread over a longer period because of the slow variation of the phase energy at this time. As seen in Fig. 5, the behaviour of the up- and downshifted plasma lines intensity as a function of the phase energy is very similar.

In order to analyse the intensity and Doppler frequency of the plasma lines, one needs an estimate of the parameters describing the ambient part of the electron distribution function (Guio et al., 1998). These parameters are estimated directly from the ion line analysis or derived from the ion line analysis. Figure 6 presents the parameters of the ambient part of the electron distribution function interpolated at the altitude corresponding to the peak of the F-region where the plasma lines are measured. The electron density $n_{e}$ and electron temperature $T_{e}$ are directly estimated. The electron collision frequency $v_{e}$ includes the electronneutral and electron-ion collision frequencies defined by (Newman and Oran, 1981)

$$
\begin{aligned}
& v_{e n}=5.4 \times 10^{-10} n_{n} T_{e}^{1 / 2} \\
& v_{e i}=\left(34.0+4.18 \log \frac{T_{e}^{3}}{n_{e}}\right) n_{e} T_{e}^{-3 / 2}
\end{aligned}
$$
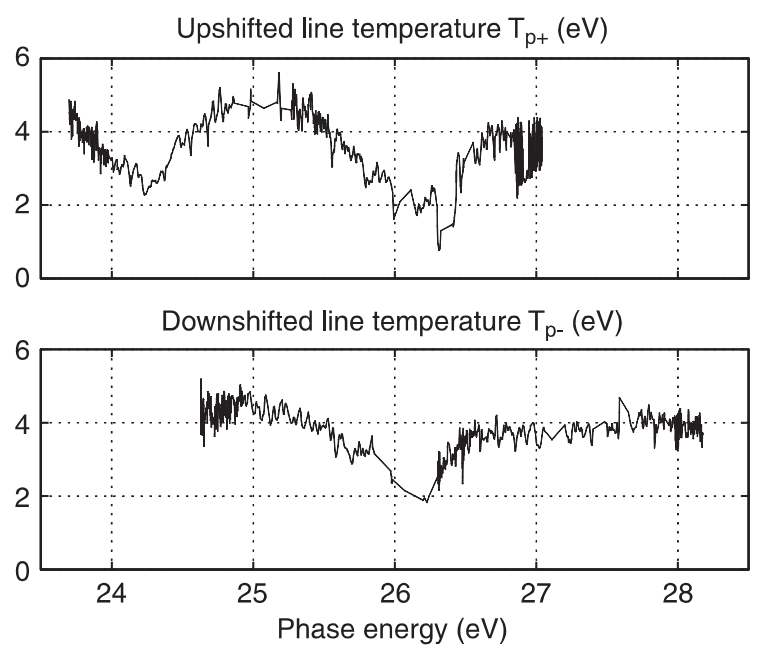

Fig. 5. The temperature $T_{p+}$ and $T_{p-}$ of the up- and downshifted plasma lines from the 1-h observation of Figs. 4 and 3 plotted as a function of their phase energy, respectively $E_{\phi+}$ and $E_{\phi-}$ 


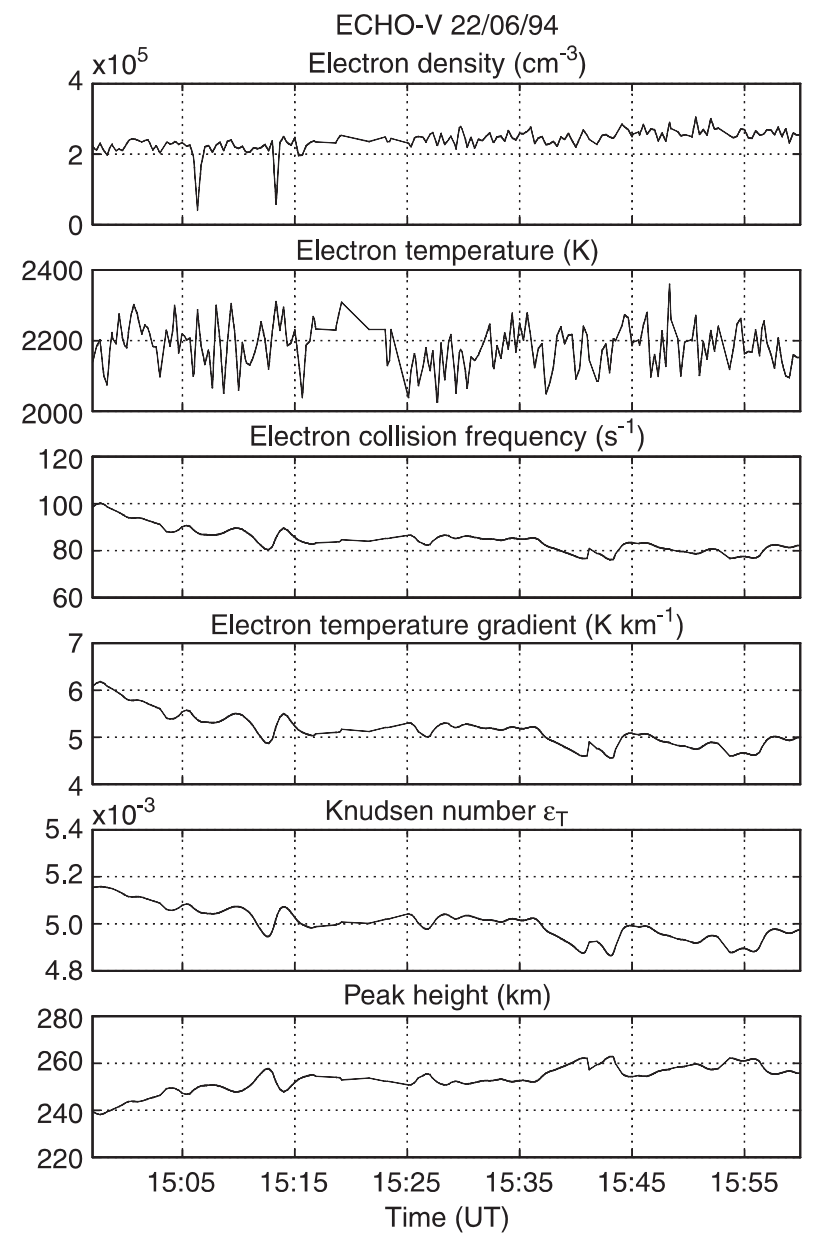

Fig. 6. The parameters for the ambient part of the electron distribution function interpolated at the altitude where the critical frequencies of the plasma lines are estimated (bottom panel)

where the density are in $\mathrm{cm}^{-3}$ and $n_{n}$ is the neutral density calculated by the MSIS-90 model (Hedin, 1991). The temperature gradient $\nabla T_{e}$ is estimated numerically from the ion line data. The Knudsen number $\epsilon_{T}=2 \lambda_{e} \nabla \log T_{e}$ (Guio et al., 1998) where $\lambda_{e}$ is the electron mean free path, is a parameter used in the Spitzer function (Cohen et al., 1950; Spitzer and Härm, 1953) to represent the electron ambient distribution function in presence of a temperature gradient.

\section{Analysis}

The analysis is split into two parts. First the electron suprathermal distribution function is adjusted so that the calculated intensity matches the measured intensity. When a satisfactory suprathermal distribution function is determined, it is then used to calculate the electron Doppler velocity $u_{e}$ as well as the plasma frequency $\omega_{e}$.

\subsection{Intensity analysis}

In order to estimate the plasma line temperature theoretically, we need a model for the suprathermal electrons. We use the suprathermal velocity distribution $f_{s}$ which we derive from the angular energy flux $\phi$ calculated by the electron transport model code along the Earth magnetic field, described in Lilensten et al. (1989) and Lummerzheim and Lilensten (1994). The ionospheric parameters $n_{e}, T_{e}$ and $T_{i}$ we used as input to the transport code are the averaged parameters of Fig. 1. The code calculates the angular energy flux for 100 altitudes between $90 \mathrm{~km}$ and $400 \mathrm{~km}$ and we get seven angular fluxes in the altitude range $236-263 \mathrm{~km}$.

Using a BGK model for the collisions, an expression for the intensity of the plasma line can be derived from the theoretical plasma line power spectrum for any arbitrary electron velocity distribution (Sheffield, 1975; Bjørnå and Trulsen, 1986) in the same way as done by Perkins and Salpeter (1965) for isotropic but not necessarily Maxwellian distribution. This new expression for the plasma line intensity expressed in terms of the two complex functions $P$ and $Z$ is valid for any arbitrary anisotropic velocity distribution that produces a stable plasma. The complex functions $P$ and $Z$ involved in the calculation can be replaced by their numerical approximations $P_{n}$ and $Z_{n}$ defined in Guio et al. (1998) and the intensity expressed as a temperature $T_{p \pm}$ of the plasma lines is written

$$
\begin{aligned}
k_{b} T_{p \pm} & =k_{b} T_{e} \times \\
& \frac{v_{\phi \pm} \operatorname{Im} P_{n}\left(\frac{v_{\phi \pm}}{v_{e}}\right)-\frac{v_{\phi \pm}}{v_{e}} \frac{v_{e}}{k_{ \pm}}\left|P_{n}\left(\frac{v_{\phi \pm}}{v_{e}}\right)\right|^{2}}{v_{e} \operatorname{Im} Z_{n}\left(\frac{v_{\phi \pm}}{v_{e}}\right)+\left(\frac{v_{e}}{v_{\phi \pm}}\right)^{2} \frac{v_{e}}{k_{ \pm}} \operatorname{Re} P_{n}\left(\frac{v_{\phi \pm}}{v_{e}}\right)}
\end{aligned}
$$

where $v_{e}$ is the sum of the electron-neutral and electronion collision frequencies of Eqs. (2) and (3), $v_{e}=\sqrt{k_{b} T_{e} / m_{e}}$ is the electron thermal velocity and

$$
\begin{aligned}
& P_{n}\left(\frac{v_{\phi}}{v_{e}}\right)=(1-\alpha) P_{n}^{a}\left(\frac{v_{\phi}}{v_{e}}\right)+\alpha P_{n}^{s}\left(\frac{v_{\phi}}{v_{e}}\right) \\
& Z_{n}\left(\frac{v_{\phi}}{v_{e}}\right)=(1-\alpha) Z_{n}^{a}\left(\frac{v_{\phi}}{v_{e}}\right)+\alpha Z_{n}^{s}\left(\frac{v_{\phi}}{v_{e}}\right)
\end{aligned}
$$

where $P_{n}^{a}, Z_{n}^{a}$ are the contributions from the ambient population while $P_{n}^{s}, Z_{n}^{s}$ are due to the suprathermal electrons. The dimensionless number $\alpha$ denotes the percentage of suprathermal electrons.

Our investigation to determine the ad hoc velocity distribution function for the suprathermal electrons shows that the detailed structure of the distribution in the concerned energy range $(23-29 \mathrm{eV})$ is depending on the solar intensity flux responsible for the creation of photo-electrons. Figure 7 shows the model for the solar intensity flux spectrum (Torr and Torr, 1985) we basically used. The solar intensity for any F10.7 is deduced by interpolation between the two reference fluxes of Fig. 7. Since that time, a lot of work has been done in order to get a better estimate of this flux. Most recent results include Warren et al. (1998a, b); Tobiska and Eparvier (1998) and Woods et al. (1998). We tested several more recent models, but in the energy range relevant in this paper, we did not find significant 


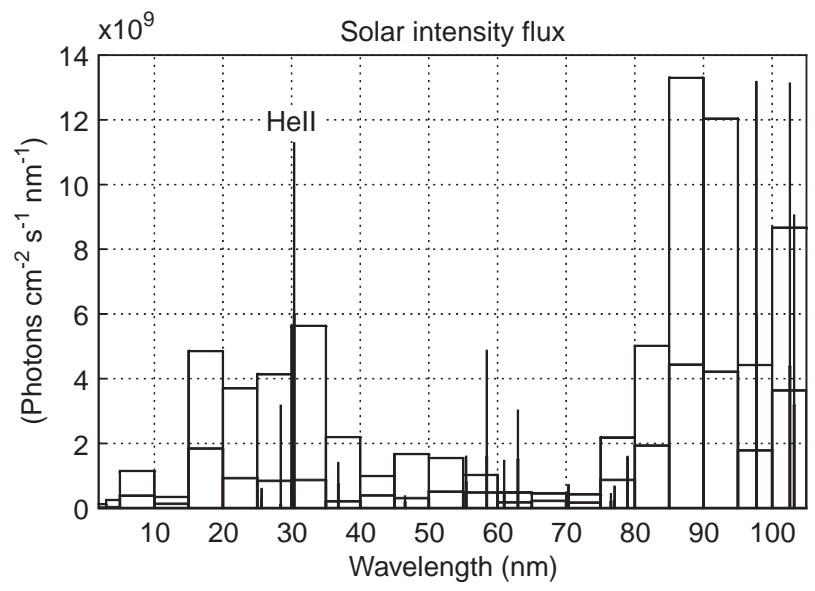

Fig. 7. The model for the solar intensity flux (Torr and Torr, 1985). The thin line is for solar maximum $(\mathrm{F} 10.7=243)$ and the thick line for solar minimum $(\mathrm{F} 10.7=68)$. The solar flux for any arbitrary F10.7 is deduced by interpolation between theses two references fluxes

differences. The cross section set is from Fennelly and Torr (1992). The two sharp peaks in the suprathermal angular velocity distribution function, seen in the top panel of Fig. 8, at energies $24.25 \mathrm{eV}$ and $26.25 \mathrm{eV}$ are the effect of the photoionisation of $\mathrm{N}_{2}$ and $\mathrm{O}$ by the discrete line of photons of wavelength $30.378 \mathrm{~nm}$ $(40.812 \mathrm{eV})$, corresponding to the solar emission of HeII in the chromospheric network and coronal holes. Indeed, ionisation thresholds are $15.58 \mathrm{eV}$ for $\mathrm{N}_{2}$ and $13.61 \mathrm{eV}$ for O. During an ionisation, the electron leaves the ion with the energy of the input photon minus the threshold energy, which should give two peaks at energies $25.232 \mathrm{eV}$ for $\mathrm{N}_{2}$ photoionisation and $27.202 \mathrm{eV}$ for $\mathrm{O}$ photoionisation. The discrepancy found can be interpreted as a mean energy left by the incident photon to the target atom or molecule during an ionization : in average, the nitrogen ion keeps about one electronvolt. This result was theoretically expected by chemists : the study of the dissociative ionisation of $\mathrm{N}_{2}$ by photons between 23 and $30 \mathrm{eV}$ has shown that the kinetic energy of the ion evolves with the initial energy of the photon (R. Thissen, personal communication). When the electron is left with no energy, the mean energy of the ion may be up to $1 \mathrm{eV}$, and even more (1.6 eV has been observed with initial photons of $30 \mathrm{eV}$ ). To our knowledge, if this interpretation and our comparison are correct it is the first time that it is conspicuous and that this energy can be numerically evaluated. It has a certain importance as far as electron density computations are concerned. Indeed, in the ionospheric models, the coefficients for the chemical recombinations involving $\mathrm{N}_{2}^{+}$are considered for a ground state ion. The coefficients involving excited ions can be multiplied by a factor of 10 (Chiu et al., 1995). The effect of excited neutral nitrogen (and especially vibrational excitation) on calculation of electron densities has long been a subject of study [see for example Pavlov and Buonsanto (1996) and references herein], but the same effect for ions is still a new subject of investigation.

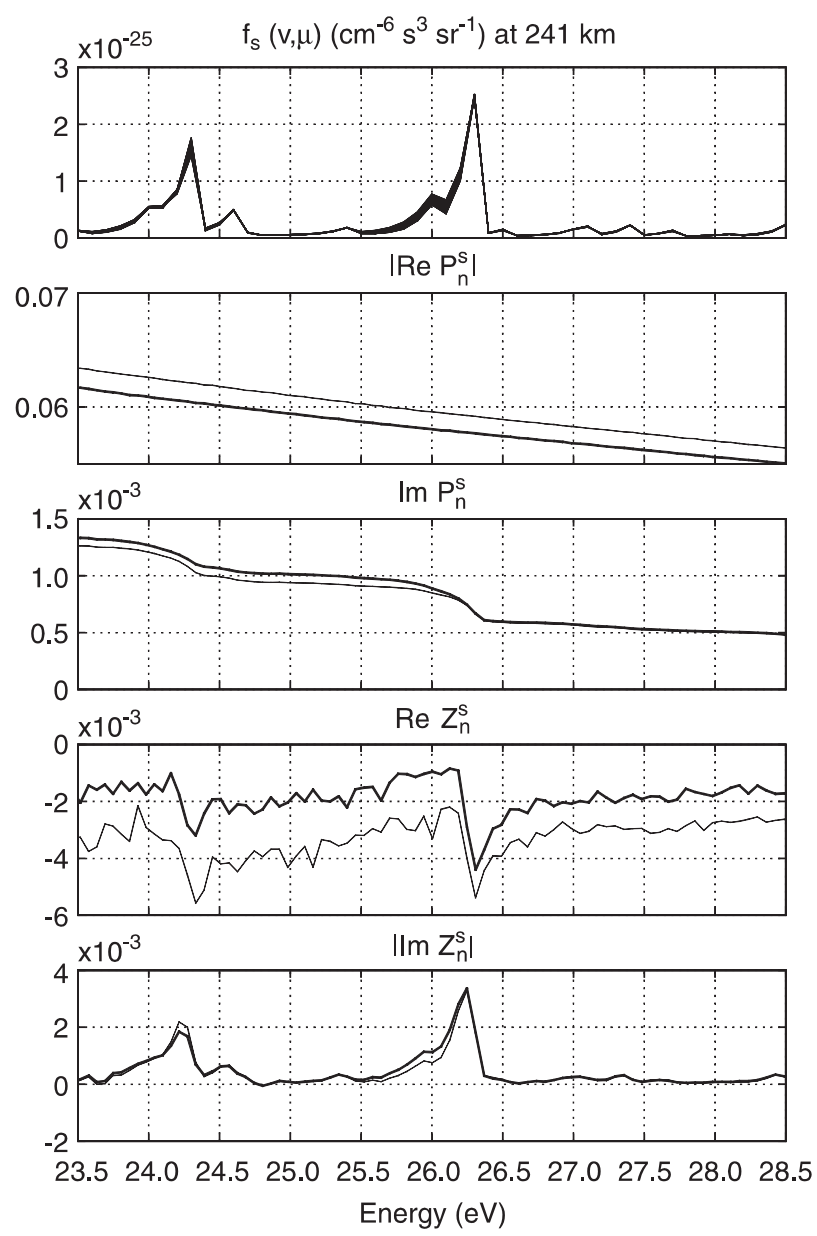

Fig. 8. The suprathermal electron angular velocity distribution function (top panel) at $241 \mathrm{~km}$ calculated by a 32-streams run of the transport code to gives the distribution at 32 angles and the complex functions $P_{n}^{s}$ and $Z_{n}^{s}$ calculated numerically with this distribution function, in the energy range $23.5-28.5 \mathrm{eV}$. The thin line is for downward phase energy and the thick line is for upward phase energy

Figure 8 shows the velocity angular distribution in the energy range $23.5-28.5 \mathrm{eV}$ (top panel) calculated by the transport code at $241 \mathrm{~km}$ and the real and imaginary parts of both the $P_{n}^{s}$ and $Z_{n}^{s}$ functions estimated with this distribution. It is seen that the two sharp peaks at $24.25 \mathrm{eV}$ and $26.25 \mathrm{eV}$ in the angular distribution (top panel) transform into the imaginary part of $Z_{n}^{s}$ function (proportional to the Landau damping) as increases at the same energy and into the imaginary part of $P_{n}^{s}$ function (proportional to the one-dimensional reduced distribution along the scattering direction) as a sharp decrease in the suprathermal population.

The magnitude of the suprathermal distribution at the energies corresponding to the two sharp peaks at $24.25 \mathrm{eV}$ and $26.25 \mathrm{eV}$ is proportional to the flux of the solar discrete emission line of HeII and so is the imaginary part of $Z_{n}^{s}$. At the same time it does not influence significantly the magnitude of the distribution at other energies. So that, we had to actually reduce by five the intensity of the flux of the HeII discrete line (see Fig. 7) for the solar minimum i.e. F10.7 $=68$, while keeping the same value for the continuum in order to get 
a magnitude of the damping that reproduced correctly the temporal variations of the plasma line temperatures. Such variations of the intensity of the flux over time period of a few hour have been observed (J. Aboudarham, personal communication).

In addition to the adjustment of the intensity of the flux of the HeII discrete line and in order to resolve correctly the two peaks at these energies we modified the energy grid of the transport model in the range 20-32 eV to a higher resolution linear grid of energy step $\cdot 1 \mathrm{eV}$.

Figure 9 shows the results of the theoretical calculations of the intensities together with the data. After the adjustments of the parameters in the transport code, the calculated intensities are seen to be in good agreement with the measured ones. It is important to note that while these modifications in the parameter of the transport code influence substantially the distribution function at the energies corresponding to the two peaks, they do not change otherwise the global properties of the suprathermal electron distribution function.

\subsection{Doppler frequency analysis}

We now present a method to derive both the electron Doppler velocity $u_{e}$ of the ambient electron population and the electron plasma frequency $\omega_{e}$ from the measured up- and down shifted plasma line frequencies.

The down- and upshifted plasma lines correspond to two Langmuir waves $\left(k_{-}, \omega_{-}\right)$and $\left(k_{+}, \omega_{+}\right)$travelling away from and toward the radar, respectively, in the backscatter geometry. They are high-frequency solutions of the dispersion relation (Guio et al., 1998)

$1+\left(\frac{\omega_{e}}{k_{ \pm} v_{e}}\right)^{2} \operatorname{Re} Z_{n}\left(\frac{2 \pi f_{r \pm}}{k_{ \pm} v_{e}}\right)=0$

Eliminating $\omega_{e}$ and $v_{e}$ between these two equations and replacing $Z_{n}$ by its ambient and suprathermal compo-
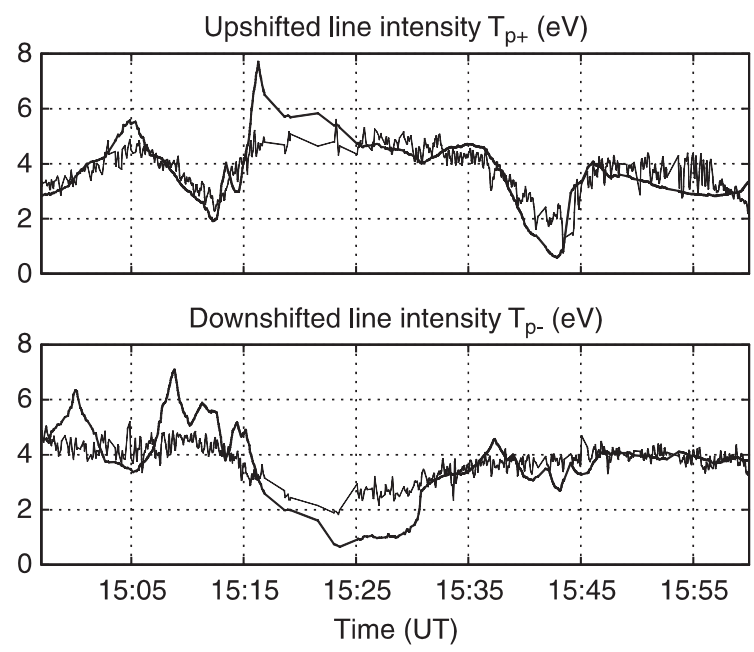

Fig. 9. Comparison between the measured plasma line intensities and the modelled intensities as a function of time. The modelled intensities are calculated using the $P_{n}^{s}$ and $Z_{n}^{s}$ functions of Fig. 8 nents and replacing the frequencies $f_{r \pm}$ by the phase velocity $v_{\phi \pm}$, the electron Doppler velocity $u_{e}$ of the ambient electron population is the solution of the following equation

$$
\begin{aligned}
& k_{+}^{2}\left((1-\alpha) \operatorname{Re} Z_{n}^{a}\left(\frac{v_{\phi-}-u_{e}}{v_{e}}\right)+\alpha \operatorname{Re} Z_{n}^{s}\left(\frac{v_{\phi-}}{v_{e}}\right)\right)= \\
& k_{-}^{2}\left((1-\alpha) \operatorname{Re} Z_{n}^{a}\left(\frac{v_{\phi+}-u_{e}}{v_{e}}\right)+\alpha \operatorname{Re} Z_{n}^{s}\left(\frac{v_{\phi+}}{v_{e}}\right)\right)
\end{aligned}
$$

where the drift velocity $u_{e}$ appears only in the terms relative to the ambient component $Z_{n}^{a}$ and we will consider here two models of velocity distribution to describe the ambient population: a Maxwellian and a Spitzer function that takes into account the gradient of the electron temperature (Guio et al., 1998).

Once the Doppler velocity $u_{e}$ is found, the plasma frequency $\omega_{e}$ is given by

$$
\begin{aligned}
\omega_{e}= & \left(k_{ \pm} v_{e}\right) \times \\
& \left|(1-\alpha) \operatorname{Re} Z_{n}^{a}\left(\frac{v_{\phi \pm}-u_{e}}{v_{e}}\right)+\alpha \operatorname{Re} Z_{n}^{s}\left(\frac{v_{\phi \pm}}{v_{e}}\right)\right|^{-\frac{1}{2}}
\end{aligned}
$$

either evaluated for the down-going Langmuir wave with phase velocity $v_{\phi \pm}$ or the up-going one with phase velocity $v_{\phi-}$.

Figures $10-12$ show the result of this analysis. Figure 10 presents the Maxwellian case whereas Fig. 11 is for the Maxwellian superimposed with the suprathermal distribution and Fig. 12 is for the Spitzer function superimposed with the suprathermal distribution.

The data set can be separated into three intervals where the Doppler velocity $u_{e}$ is constant: before the first
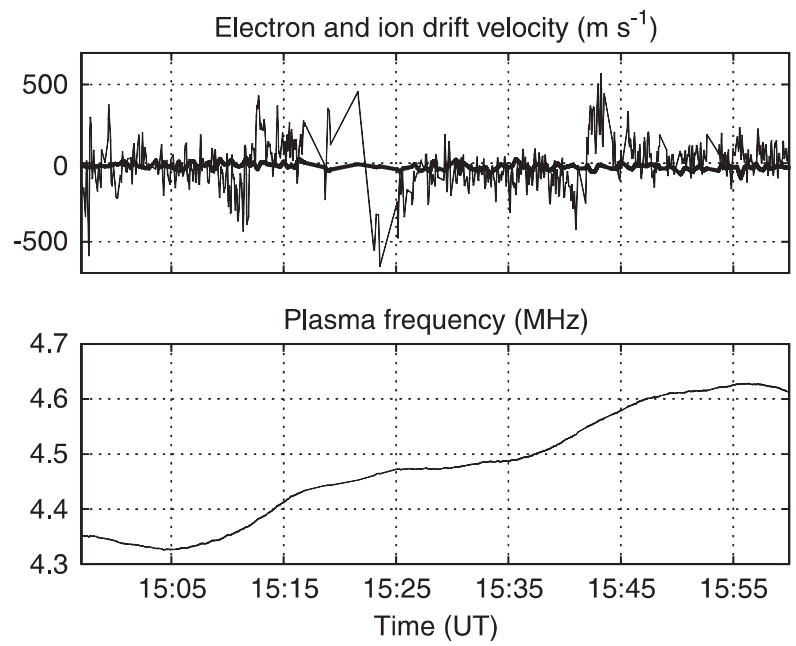

Fig. 10. The upper panel shows the estimated ion drift velocity $u_{i}$ (thick line) from the ion line analysis and the electron drift velocity $u_{e}$ (thin line) from the analysis method based on Eq. (8) without suprathermal population $(\alpha=0)$ and assuming a Maxwellian ambient population with the parameters of Fig. 6 . The lower panel shows the estimated plasma frequency $\omega_{e} / 2 \pi$ given by Eq. (9) 

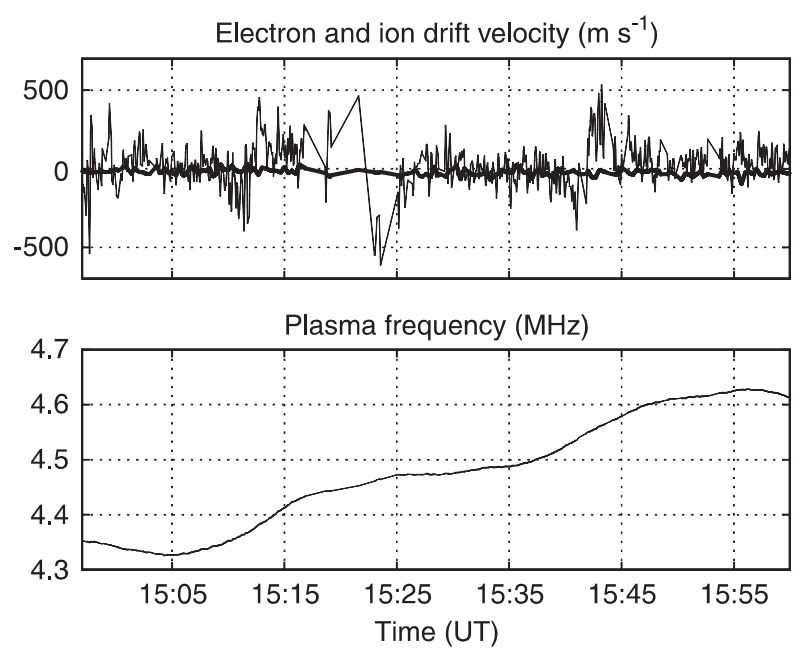

Fig. 11. The upper panel shows the estimated ion drift velocity $u_{i}$ (thick line) from the ion line analysis and the electron drift velocity $u_{e}$ (thin line) from the analysis method based on Eq. (8) with a suprathermal population described by the distribution function of Fig. 8 with a Maxwellian background of parameters of Fig. 6. The lower panel shows the estimated plasma frequency $\omega_{e} / 2 \pi$ given by Eq. (9)
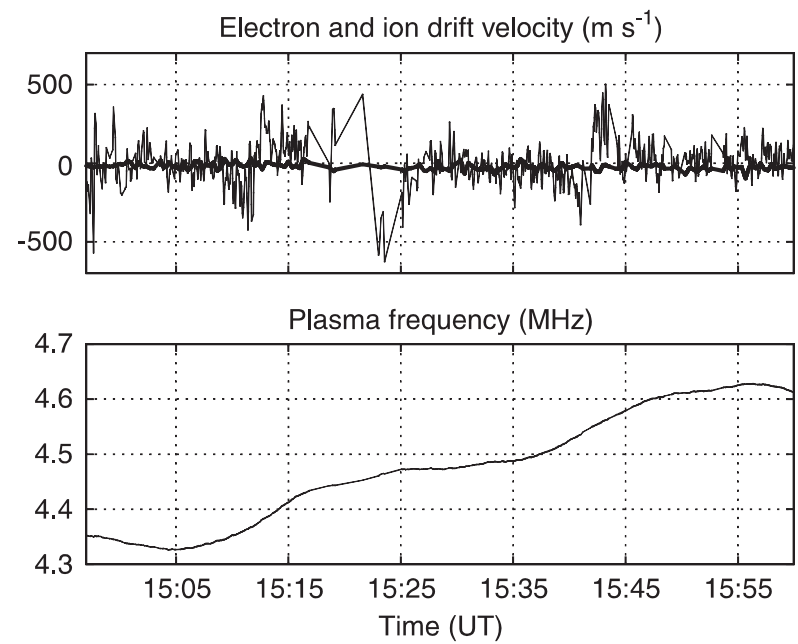

Fig. 12. The upper panel shows the estimated ion drift velocity $u_{i}$ (thick line) from the ion line analysis and the electron drift velocity $u_{e}$ (thin line) from the analysis method based on Eq. (8) with a suprathermal population described by the distribution function of Fig. 8 and a Spitzer function for the ambient electrons with parameters of Fig. 6 . The lower panel shows the estimated plasma frequency $\omega_{e} / 2 \pi$ given by Eq. (9)

feature of change of sign at 15:12 UT, between the second feature at 15:23 UT and the third one at 15:43 UT and after this last one. Table 1 gives the mean value of the Doppler velocity $u_{e}$ for the different models considered as well as the mean value of the ion Doppler velocity $u_{i}$ for these three time intervals. The effect of the suprathermal electrons is clearly seen, our suprathermal velocity distribution reduces the relative differences of $u_{e}$ between the three intervals. The effect of the Spitzer function is thereafter to shift down the whole mean drift velocity, which we expected as the heat
Table 1. Mean value of the electron Doppler velocity $u_{e}$ for the four different models considered: a Maxwellian, a Spitzer function, a Maxwellian superimposed with a suprathermal distribution and a Spitzer superimposed with a suprathermal distribution as well as the ion Doppler velocity $u_{i}$ estimated from the ion line analysis. The suprathermal distribution is the one shown in Fig. 8 and the parameters of the Spitzer function are the one of Figure 6. The mean Doppler are calculated for the three time intervals considered. The first number in each column is the velocity in $\mathrm{ms}^{-1}$ while the number in parenthesis is the equivalent frequency Doppler shift in $\mathrm{Hz}$

\begin{tabular}{llll}
\hline & $14: 57-15: 09$ & $15: 26-15: 39$ & $15: 46-16: 00$ \\
\hline Maxwellian & $-25(-74)$ & $-72(-214)$ & $+37(+112)$ \\
Spitzer & $-57(-169)$ & $-95(-282)$ & $+17(+50)$ \\
Maxwellian + Supra & $+22(+66)$ & $-10(-29)$ & $+48(+143)$ \\
Spitzer + Supra & $-10(-30)$ & $-32(-97)$ & $+27(+82)$ \\
Ion line Doppler $u_{i}$ & $-15(-45)$ & $-27(-81)$ & $-29(-86)$ \\
\hline
\end{tabular}

flow effect behaves like a mean Doppler velocity. The superposition of the suprathermal distribution to the Spitzer function (with parameters of Fig. 6) gives estimates of $u_{e}$ in best agreement with $u_{i}$.

On the other hand, we were not able to reduce considerably the features at 15:12 UT, 15:24 UT and 15:43 UT even though they are probably the effect of the suprathermal electrons as it can be thought by examining carefully the real part of the $Z_{n}^{s}$ function of Fig. 8 . Qualitatively, the real part of $Z_{n}^{s}$ around $24.25 \mathrm{eV}$ and $26.25 \mathrm{eV}$ has a correct shape to compensate these changes in the electron Doppler velocity $u_{e}$. These three events are characterised by an abrupt change of sign of the Doppler velocity. During the first feature at 15:12 UT the Doppler velocity first decreases, then increases. At this time, the phase energy of the upshifted plasma line moves across $24.25 \mathrm{eV}$ where the real part of $Z_{n}^{s}$ first increases, then decreases while the phase energy of the downshifted plasma line is around $25.25 \mathrm{eV}$ where the real part of $Z_{n}^{s}$ increases slowly (see Fig. 8). Thus the term $k_{-}^{2} \operatorname{Re} Z_{n}^{s}\left(E_{\phi+}\right)$ of Eq. (8) is varying qualitatively correctly so that Eq. (8) should be satisfied without such variations being reflected on the electron Doppler velocity $u_{e}$. During the two other features the same effect is observed. At 15:24 UT the phase energy of the downshifted plasma line moves across $26.25 \mathrm{eV}$ which gives an opposite effect, it is now the term $k_{+}^{2} \operatorname{Re} Z_{n}^{s}\left(E_{\phi-}\right)$ of Eq. (8) that compensates. At 15:43 UT, the phase energy of the upshifted plasma line moves across $26.25 \mathrm{eV}$ which gives an effect in the same direction as the first one. Moreover the first feature at 15:12 UT is not so emphasised as the two other ones. It corresponds to the $\mathrm{N}_{2}$ photoionisation peak while the two other features correspond to the $\mathrm{O}$ photoionisation which effect on the real part of $Z_{n}^{s}$ is more emphasised than for the $\mathrm{N}_{2}$ peak.

It can be pointed out that the frequency correction caused by our model of photoelectrons is of the same order as the one predicted with a Maxwellian photoelectron population (Bauer et al., 1976). We note also that while the difference of the electron mean velocity $u_{e}$ is substantial, the difference in the estimated plasma frequency $\omega_{e}$ remains under $1 \mathrm{KHz}$. 


\section{Conclusion}

We have discussed a high time resolution plasma line data set measured by the EISCAT VHF radar in the summer daytime ionosphere. We have presented a method to analyse both the intensity and the Doppler frequency shift of the plasma lines using a model taking into account the suprathermal electron population and the effect of the electron temperature gradient on the ambient population. The data set has been analysed with this method. Parameters of the electron transport code, such as the solar intensity flux and the ionisation threshold of $\mathrm{N}_{2}$ and $\mathrm{O}$ had to be adjusted to take into account correctly the detailed structures of the suprathermal distribution in the energy range $23.5-28.5 \mathrm{eV}$. Our model reproduces well the temporal variations of the intensity of the plasma lines and we have identified the effect of $\mathrm{N}_{2}$ and $\mathrm{O}$ photoionisation at $24.25 \mathrm{eV}$ and $26.25 \mathrm{eV}$ caused by the solar discrete emission line HeII. We then derived the electron mean Doppler velocity and the plasma frequency and identified also the effect of $\mathrm{N}_{2}$ and $\mathrm{O}$ photoionisation. It is shown that our model for photoelectrons superimposed with a Spitzer function for the ambient electrons is able to reproduce the variations in the intensity of the plasma lines as well as explain substantially the variations of the Doppler frequency of the plasma lines for the data set measured with the EISCAT VHF radar.

Acknowledgements. The authors would like to thank Odile Dutuit de Lure and Roland Thissen for helpful discussions as well as the EISCAT personal for their efforts in making the radar available. EISCAT is funded by the CNRS of France, the SA of Finland, the MPG of Germany, the NFR of Norway, the NFR of Sweden, and the PPARC of United Kingdom.

Topical Editor D. Alcaydé thanks two referees for their help in evaluating this paper.

\section{References}

Bauer P., K. D. Cole, and G. Lejeune, Field-aligned electric currents and their measurement by the incoherent backscatter technique, Planet. Space Sci., 24, 479-485, 1976.

Bjørnå N., and J. Trulsen, Effect of a power law particle flux on the ionospheric incoherent scattering cross section, Phys. Scr., 33, 284-288, 1986

Bjørnå, N., O. Havnes J. O. Jensen, and J. Trulsen, Enhancement of the incoherent scattering plasma lines due to precipitating protons and secondary electrons, Phys. Scr., 25, 632-636, 1982.

Chiu, Y.-H., H. Fu, J.-T. Huang, and S. L. Anderson, Vibrational mode effects, scattering dynamics, and energy disposal in reaction of $\mathrm{C}_{2} \mathrm{H}_{2}^{+}$with methane, J. Chem. Phys., 102, 1199 1216, 1995.

Cohen R. S., L. Spitzer, and P. McR. Routly, The electrical conductivity of an ionized gas, Phys. Rev., 80, 230-238, 1950.

Fennelly J. A., and D. G. Torr, Photoionization and photoabsorption cross sections of $\mathrm{O}, \mathrm{N} 2$ and $\mathrm{N}$ for aeronomic calculations, Atomic Data and Nuclear data tables, 51, 321-363, 1992.

Guio P., N. Bjørnå, and W. Kofman, Alternating-code experiment for plasma-line studies. Ann. Geophys., 14, 1473-1479, 1996.

Guio P., J. Lilensten , W. Kofman, and N. Bjørnå, Electron velocity distribution function in a plasma with temperature gradient and in the presence of suprathermal electrons: application to incoherent scatter plasma lines, Ann. Geophys., 16, 1226-1240, 1998.

Hagfors T., and M. Lehtinen, Electron temperature derived from incoherent scatter radar observations of the plasma line frequency, J. Geophys. Res., 86, 119-124, 1981.

Hedin A. E., Extension of the MSIS Thermospheric Model into the Middle and Lower Atmosphere, J. Geophys. Res., 96, 11591172, 1991.

Heinselman C. J., and J. F. Vickrey, On the spectral analysis and interpretation of incoherent scatter plasma line echoes, Radio Sci., 27, 221-230, 1992.

Kirkwood S., H. Nilsson, J. Lilensten, and M. Galand, Strongly enhanced incoherent-scatter plasma lines in aurora, J. Geophys. Res., 100, 21343-21355, 1995.

Kofman W., and G. Lejeune, Determination of low energy photoelectron distribution from plasma line measurements at Saint Santin, Planet. Space Sci., 28, 661-673, 1980.

Kofman W., G. Lejeune, T. Hagfors, and P. Bauer, Electron temperature measurements by the plasma line technique at the french incoherent scatter radar facilities, J. Geophys. Res., 86, 6795-6801, 1981.

Kofman W., J.-P. St-Maurice, and A. P. van Eyken, Heat flow effect on the plasma line frequency, J. Geophys. Res., 98, 6079$6085,1993$.

Lejeune G., and W. Kofman, Photoelectron distribution determination from plasma line intensity measurements obtained at Nanay (france), Planet. Space Sci., 25, 123-133, 1977.

Lilensten J., W. Kofman, J. Wisenberg, E. S. Oran, and C. R. Devore, Ionization efficiency due to primary and secondary photoelectrons: a numerical model, Ann. Geophys., 7, 83-90, 1989.

Lummerzheim D., Electron transport and optical emission in the aurora, PhD thesis, University of Alaska, Fairbanks, 1987.

Lummerzheim D., and J. Lilensten, Electron transport and energy degradation in the ionosphere: evaluation of the numerical solution, comparison with laboratory experiments, auroral observations, Ann. Geophys., 12, 1039-1051, 1994.

Newman A. L., and E. S. Oran, The effects of electron-neutral collisions on the intensity of plasma lines, J. Geophys. Res., 86, 4790-4794, 1981.

Oran E. S., V. B. Wickwar, W. Kofman, and A. Newman, Auroral plasma lines: a first comparison of theory and experiment, J. Geophys. Res., 86, 199-205, 1981.

Pavlov A. V., and M. J. Buonsanto, Using steady state vibrational temperature to model effects of $\mathrm{N}_{2}^{*}$ on calculations of electron densities, J. Geophys. Res., 101, 26941-26945, 1996.

Perkins F., and E. E. Salpeter, Enhancement of plasma density fluctuations by nonthermal electrons, Phys. Rev. A, 128, 55-62, 1965.

Perkins F., E. E. Salpeter, and K. O. Yngvesson, Incoherent scatter from plasma oscillations in the ionosphere, Phys. Rev. Lett., 14, 579-581, 1965.

Sheffield J., Plasma scattering of electromagnetic radiation, Academic Press, New York, 1975.

Showen R. L., The spectral measurement of plasma lines, Radio Sci., 14, 503-508, 1979.

Spitzer L. Jr, and R. Härm, Transport phenomena in a completely ionized gas, Phys. Rev., 89, 977-981, 1953.

Tobiska W. K., and F. G. Eparvier, EUV97: improvements to EUV irradiance modeling in the soft X-Rays and FUV, Solar Phys., 177, 147-159, 1998.

Torr M. R., and D. G. Torr, Ionization frequencies for solar cycle 21: Revised, J. Geophys. Res., 90, 6675-6678, 1985.

Vidal-Madjar D., W. Kofman, and G. Lejeune, Mesures de la raie de plasma par diffusion incohérente à Nançay, et premiers résultats morphologiques, Ann. Geophys., 31, 227-234, 1975.

Warren H. P., J. T. Mariska, and J. Lean, A new reference spectrum for the EUV irradiance of the quiet Sun 1. Emission measure formulation, J. Geophys. Res., 103, 12077-12089, 1998a. 
Warren H. P., J. T. Mariska, and J. Lean, A new reference spectrum for the EUV irradiance of the quiet Sun 2. Comparison with observations and previous models, J. Geophys. Res., 103, 12091-12102, 1998b.

Woods T. N., G. J. Rottman, S. M. Bailey, S. C. Solomon, and J. R. Worden, Solar extreme ultraviolet irradiance measurements during solar cycle 22, Solar Phys., 177, 133-146, 1998.
Yngvesson K. O., and F. W. Perkins, Radar Thomson scatter studies of the photoelectrons in the ionosphere and Landau damping, J. Geophys. Res., 73, 97-100, 1968. 